\title{
Surgical surprises in thyroidectomy
}

\section{Introduction}

Surgical excision of the thyroid gland may be otherwise defined as dissection of recurrent laryngeal nerves (RLN). Injury to RLN is one of the most dreaded complication that we encounter in our practise. Though the RLN has a fixed anatomical location in the tracheo oesophageal grove this is not the rule always. The 2 most common deviations from normal anatomy in RLN are non recurrent laryngeal nerve(NRLN) and Galen's anastomosis(GA).

\section{Galen's anatomises-literature review}

Recent studies stated that GA as the dorsal communication between the RLN and the internal laryngeal nerve and it is present over the dorsal surface of the posterior cricoarytenoid, oblique and transverse arytenoids muscles under mucosa of hypopharynx. ${ }^{1}$ Despite various studies conducted, the prevalence of GA continues to be controversial. The study 'Galen's anastomosis-revisited, ${ }^{2}$ aimed to know the incidence of GA and its role in laryngeal muscle innervations and morphometric contribution in the formation of GA. The study included 50 adult cadaveric en-bloc laryngeal specimens. ${ }^{3}$ The branches of recurrent laryngeal and internal laryngeal nerve to the laryngeal muscles were traced and studied with a aid of digital microcalliper. During analysis, GA was found in 81/100 hemilarynges with no statistical difference between right and left side. GA was located on the dorsal surface of transverse and oblique arytenoids and posterior arytenoids muscles formed by the union of inferior descending branch of internal laryngeal nerve and posterior branch of recurrent laryngeal nerve after giving muscular branches to the larynx. ${ }^{4}$ It presents as three different patterns with the most frequent pattern being single trunk anastomosis $(71 \%)$ and the other two being double pattern $(5 \%)^{5}$ and plexus formation anastomosis $(5 \%)$. There were no statistical difference in the incidence between right and left side in all three patterns.

The study also analysed the morphometric importance of GA which is unique to this study. The diameters of the internal and recurrent laryngeal nerves measured and compared after which they had given muscular branches to form GA. The average diameter of internal laryngeal nerve is $1.27 \mathrm{~mm}$ on left side and $1.28 \mathrm{~mm}$ on right side (after giving muscular branches). The average diameter of recurrent laryngeal nerve was $0.68 \mathrm{~mm}$ on left side and $0.65 \mathrm{~mm}$ on right side (after giving muscular branches). The comparison showed statistically significant difference ( $p$-value $\leq 0.001$ ) between internal and recurrent laryngeal nerve stating internal laryngeal nerve providing greater contribution to the connection. This differs from what Dilworth who says that Galen's anastomosis may be looked on as a continuation of the Inferior laryngeal nerve upwards. It was observed that posterior cricoarytenoid is the only muscle supplied by GA. GA also provides branches to the pharynx mucosa. The study did not elicit the motor or sensory innervations. Some persistent activity following vocal cord paralysis suggests the presence of regenerate nerve supply from Galen's anastomosis. ${ }^{6,7}$ Even though it cannot always be possible to identify GA during dissection, the incidence of $81 \%$ suggests the constant presence of GA as an anatomical entity.

\author{
Special Issue - 2018 \\ Dhalapathy Sadacharan, Sankaran \\ Muthukumar, Marappan Sureshkumar \\ Department of Endocrine Surgery, Madras medical college, India
}

Correspondence: Dhalapathy Sadacharan, Associate Professor, Department of Endocrine Surgery, Madras medical college, Chennai, India, Email drsdhalapathy@gmail.com

Received: April 18, 2018| Published: November 15, 2018

\section{Non recurrent laryngeal nerve}

The NRLN is a rare anatomic variation which arises from vagus nerve with a straight course to larynx. It is common in right side and is associated with abnormal subclavian artery (arteria Lusoria). The incidence of NRLN is $0.28 \%$ to $0.52 \%$ (right side being common- $-0.3 \%$ to $0.8 \%$ and left side $-0.004-0.04 \%)^{8-9}$ and the rate of intraoperative injury is $12.9 \%$ compared to only $3-7 \%$ incidence of RLN injury. Since the rate of NRLN damage is significantly higher than that of RLN, the preoperative prediction of NRLN improves surgical technique in identifying NRLN during surgery.The incidence of NRLN is not significantly associated with patient's age, sex, nature of tumor and nodule and the use of monitor.

\section{Classification of NRLN}

Type I-origin at the level above laryngo-tracheal junction and descends with superior thyroid vessels. Type II- origin at the level of thyroid isthmus and ascends over inferior thyroid artery into the throat. Type III-nerve descends inferiorly to inferior thyroid artery trunk or branches and then ascends back into throat. ${ }^{10}$ The anatomical approach used for all the patients who underwent surgery remains same irrespective of NRLN variation. If the RLN was not found in the tracheoesophageal groove, the exploration proceeds from usual entering point of RLN to the direction of carotid sheath where a silver white cord-like structure if present which arises from vagus nerve was confirmed as NRLN. In a study, ${ }^{11} 12,083$ RLNs were identified, of which 28 were identified as NRLN. All 28 cases were found to be on right side. Of the 28 cases ( 5 men and 23 women), 4 cases of NRLN injury occurred and the injury rate was $14 \%$. For patients with nerve damage, an intraoperative end to end primary anastomosis, local seal with glucocorticoid injection and postoperatively neurotrophic drugs can be administered.

Because of the incidence of high rate of NRLN injury, preoperative diagnosis /suspiciousness of the presence of RLN is of utmost importance in preventing NRLN injury. Chest x-ray and Doppler neck might reveal widened mediastinum, compressed trachea, abnormal right subclavian artery (right NRLN) and situs inversus (left NRLN). Some clinical symptoms pointing to presence of NRLN are dysphagia (compression of oesophagus between arteria lusoria and trachea) and hoarseness of voice (because NRLN prone to easy compression by even small thyroid nodule). ${ }^{12}$ Routine identification and proper dissection of RLN during surgery is necessary to effectively reduce the rate of NRLN injury. 


\section{Recommendations}

Some basic principles should be followed in all cases to prevent nerve injury.

i) Complete dissection of the nerve to its course may identify its anatomic variation.

ii) Surgical separation should start at the margin of gland. Bunched vessel ligation and division should be avoided.

iii) Superior pole pedicle should be ligated close to gland margin to avoid injury to type I NRLN.

iv) If the RLN is not found in the normal location, search for NRLN should occur in carotid sheath and cervical vagus nerve for its course.

v) Blind hemostasis with a haemostat should be avoided.

vi) Intra operative neuro monitoring can be used if available for NRLN identification. ${ }^{13,14}$

\section{Conclusion}

The presence of NRLN and GA greatly increases the rate of risk and nerve damage because of its anatomic variation and difficult dissection. Therefore, surgeons should pay more attention in eliciting proper clinical history, preoperative assessment and meticulous and careful dissection during surgery to avoid damage to RLN.

\section{Acknowledgments}

None.

\section{Conflict of interest}

None.

\section{References}

1. Naidu L, Lazarus L, Partab P, et al. Laryngeal nerve "anastomoses". Folia Morphol (Warsz). 2014;73(1):30-36.
2. Naidu L, Lelika Ramsaroop, Prawesh Partab. Galen's "anastomosis" revisited. Clin Anat. 2012.

3. Williams AF. The nerve supply of the laryngeal muscles. J Laryngol Otol. 1951;65(5):343-348.

4. Henry BM, Pękala PA, Sanna B, et al. The anastomoses of the recurrent laryngeal nerve in the larynx: A meta-analysis and systematic review. $J$ Voice. 2016;31(4):495-503.

5. https://journals.viamedica.pl/folia morphologica/article/download/ FM.2014.../26569.

6. Sanudo JR, Maranillo E, Leon X, et al. An anatomical study of anastomoses between the laryngealnerves. Laryngoscope. 1999;109(6):983-987.

7. Mahmodlou R, Aghasi MR, Sepehrvand N. Identifying the nonrecurrent laryngeal nerve: Preventing a major risk of morbidity during thyroidectomy. Int J Prev Med. 2013;4(2):237-240.

8. Dolezel R, Jarosek J, Hana L, et al. Clinical relevanceand surgical anatomy of non-recurrent laryngeal nerve: 7 year experience. Surg Radiol Anat. 2015;37(4):321-325.

9. Fellmer PT, Bohner H, Wolf A, et al. Aleft nonrecurrent inferior laryngeal nerve in a patient withright-sided aorta, truncus arteriosus communis, and an aberrant left innominate artery. Thyroid. 2008;18(6):647-649.

10. Qiao N. Otolaryngol Head Neck Surg. 2017

11. Toniato A, Rubello D, Merante Boschin I. Non recurrent andipsilateral recurrent inferior laryngeal nerves. Minerva Chir. 2012;67:286-287.

12. Kamani D, Potenza AS, Cernea CR, et al. The nonrecurrent laryngeal nerve: anatomic and electro physiologic algorithm for reliable identification. Laryngoscope. 2015;125(2):503-508.

13. Chandrasekhar SS, Randolph GW, Seidman MD, et al. Clinical practice guideline: improving voice outcomes after thyroid surgery. Otolaryngol Head Neck Surg. 2013;148(6):1-37.

14. Donatini G, Carnaille B, Dionigi G. Increased detection of non-recurrent inferior laryngeal nerve (NRLN) during thyroid surgery using systematic intra operative neuro monitoring (IONM). World J Surg. 2013;37(1):9193. 